\title{
Fuzzy Logic Controlled Miniature LEGO Robot for Undergraduate Training System
}

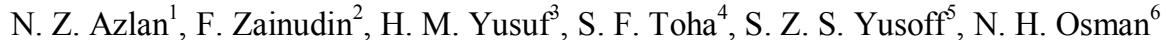 \\ Department of Mechatronics, Faculty of Engineering, International Islamic University Malaysia, \\ Jalan Gombak, 53100 Kuala Lumpur, Malaysia \\ ${ }^{1}$ sinnira@iiu.edu.my, ${ }^{2}$ zfadzilla@,iiu.edu.my, ${ }^{3}$ myhazlina@,iiu.edu.my, ${ }^{4}$ tsfauziah@iiu.edu.my, \\ syedzaini@mdx.com.my, ${ }^{6}$ hayani@yahoo.com
}

\begin{abstract}
Fuzzy logic enables designers to control complex systems more effectively than traditional approaches as it provides a simple way to arrive at a definite conclusion upon ambiguous, imprecise or noisy information. In this paper, we describe the development of two miniature LEGO robots, which are the line following and the light searching mobile robots to provide a better understanding of fuzzy logic control theory and real life application for an undergraduate training system. This study is divided into two parts. In the first part, an object sorter robot is built to perform pick and place task to load different colour objects on a fuzzy logic controlled line following robot which then carries the preloaded objects to a goal by following a white line. In the second part, an intelligent fuzzy logic controlled light searching robot with the capability to navigate in a maze is developed. All of the robots are constructed by using the LEGO Mindstorms kit. Interactive $C$ programming language is used to program fuzzy logic robots. Experimental results show that the robots has successfully track the predefined path and navigate towards light source under the influence of the fuzzy logic controller; and therefore can be used as a training system in undergraduate fuzzy logic class.
\end{abstract}

\section{INTRODUCTION}

Fuzzy logic was initiated in the 1960 s by Lofti Zadeh, a graduate student at Columbia University [1]. It is exemplified by the use of linguistic variables to represent part of the range of an ordinary crisp variable. It allows a better modeling and control of real world nonlinear and complex problems more effectively.

Teaching the concepts of fuzzy logic in the class alone might be inadequate as students may not see the real world implementation of the subject. They need an overview of fuzzy logic in engineering practice and exposure to the environment that the subject is applied [2]. Problem based approach and robots have been shown by a number of researchers to be motivating and beneficial in science and technology education [3]. Amongst the robots that are easy to be constructed and widely used in the university nowadays is the Lego robot. The power, ease of use, and small size of Lego is found to be appealing to the students since it provides an excellent medium to promote creativity, design and programming skill [4].

Therefore, in this paper we propose a fuzzy logic based line following robot with a sorter arm and a light searching robot with the ability to navigate in a maze as a part of the training system to motivate and help undergraduate students in the university to understand the underlying concepts of fuzzy logic better. The hardware design of the robots is described in section II of this paper while the developed fuzzy logic controller is represented in section III. Section IV discusses the experimental results of this project and finally conclusions are drawn in section $\mathrm{V}$.

\section{HARDWARE DESIGN}

\section{A. Fuzzy Logic Line Follower Robot and Sorter Robot Hardware Design}

The hardware design of the robotic system is divided into two main parts. The first part focuses on the development of a line following robot while the second part concentrates on the object sorting robot. Both robots are built by using the components, i.e. bricks, gears, motors and sensors provided in the LEGO Mindstorms set. The LEGO Mindstorms set makes ideas about robotics accessible to anyone even those without sophisticated mechanical-engineering skills to build computer-controlled mechanism by simply snapping parts together [5].

The object sorting robot is essentially a long robotic arm that is attached to a stationary base. The sorter could swivel around, able to pick up small objects and place them in different places. It sorts objects based on their colours into two piles, one pile for black coloured items and another pile for lighter coloured items. The sorted items are then loaded onto two different locations (left and right) on the line following robot. The flow chart of the operation of this robot is illustrated in Fig. 1. One Hitachi H8 series (RCX) is used to act as the brain of the sorting robot. As to differentiate the object colours, one light sensor is utilized. Two motors are used to provide its rotational motion, in both vertical as well as horizontal directions. The hardware design of this sorter robot arm is illustrated as in Fig. 2.

After the sorting process, the line following robot carries the sorted objects to two different locations by following a white line that is attached to a black background. This robot is controlled by fuzzy logic controller. Two sensors are attached beneath the robot base to recognize the white line. The input value from the sensors is a gradient. A touch sensor is used for the purpose of detecting any obstacles and stopping the robot while tracking the white line. Two motors 
are utilized to drive its left and right wheels. Different from the sorter robot arm, this robot is programmed by using the Handy Board, which is based on the Motorola 68HC11 microprocessor as shown in Fig. 3, due to the limitation of the RCX in implementing the fuzzy logic control. The hardware construction of this line following robot is illustrated in Fig. 4.

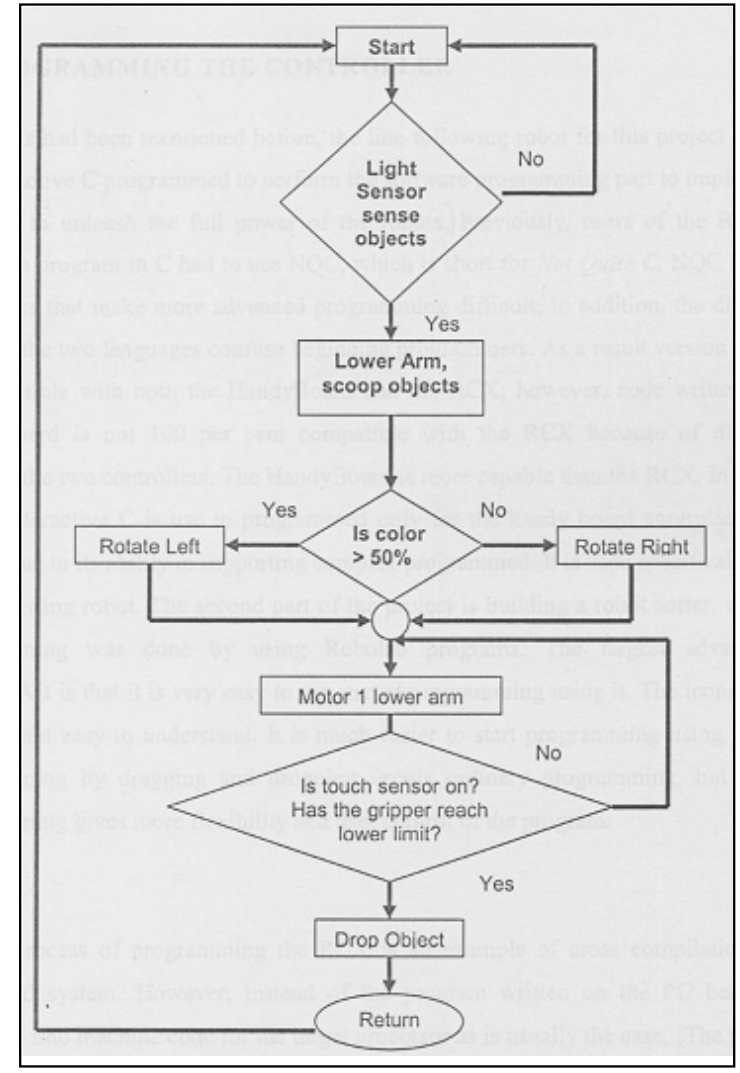

Fig. 1 Flow Chart of the Sorter Robot Operation

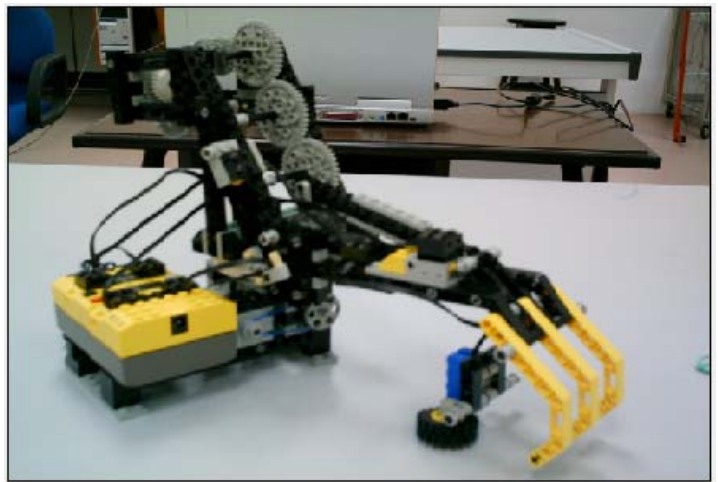

Fig. 2 Object Sorter Robot

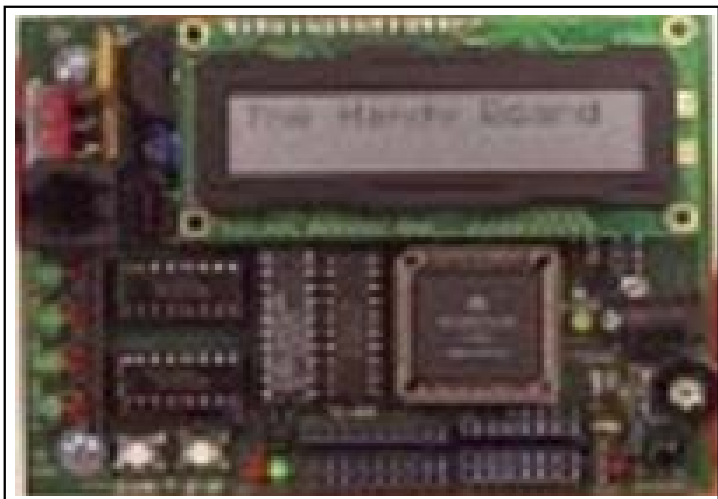

Fig. 3 Handyboard

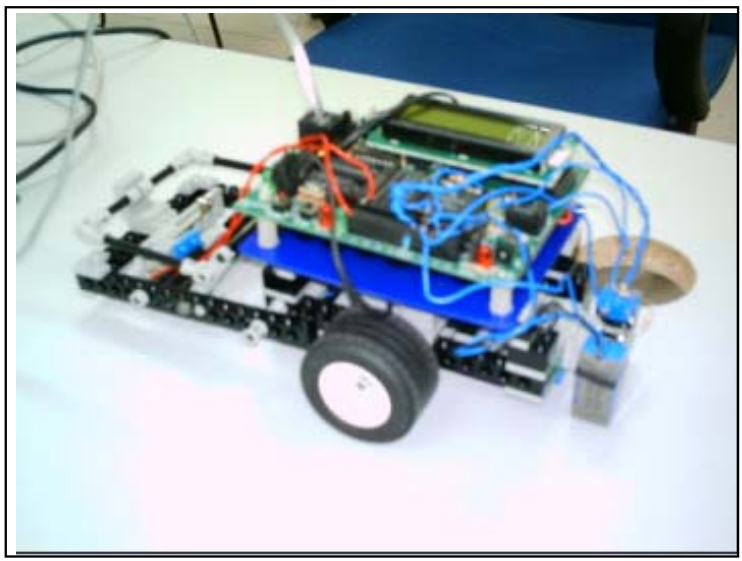

Fig. 4 Line Following Robot

\section{B. Fuzzy Logic Light Searching Robot Hardware Design}

Similar to the line following robot, the hardware part of the light searching robot including the bricks, plates, beams, motors and sensors are also constructed from the LEGO Mindstorms set. The two light sensors of the robot are used to find the brightest spot in a labyrinth and its two touch sensors sense the walls for collision avoidance. As for the microcontroller, the handyboard is used to program the fuzzy logic algorithm. It receives the readings from both the left and right light sensors which then serve as inputs to the fuzzy control system. Then, based on the rules set, the fuzzy logic controller determines the appropriate output voltage, which directly influences the speed of the right and left motor. This process guides and maintains the robot to find the path to reach the point with the highest intensity of light. Besides that, the robot also is desired to be able to navigate inside the maze without colliding with the walls. The final design of the robot is illustrated as in Fig. 5 and the operational sequence of this robot is shown in Fig. 6. 


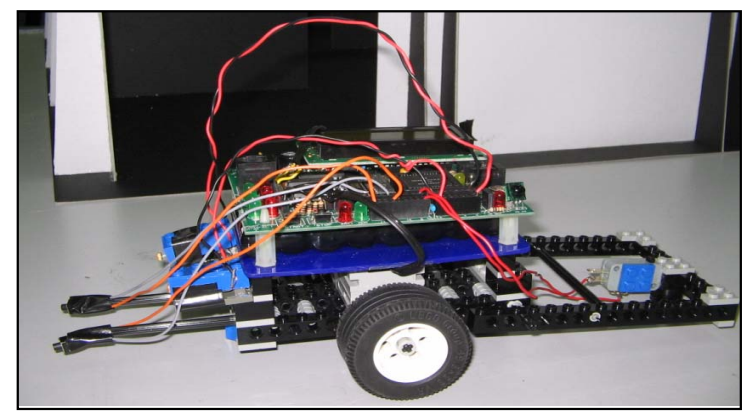

Fig. 5 Final Design of Light Search Robot

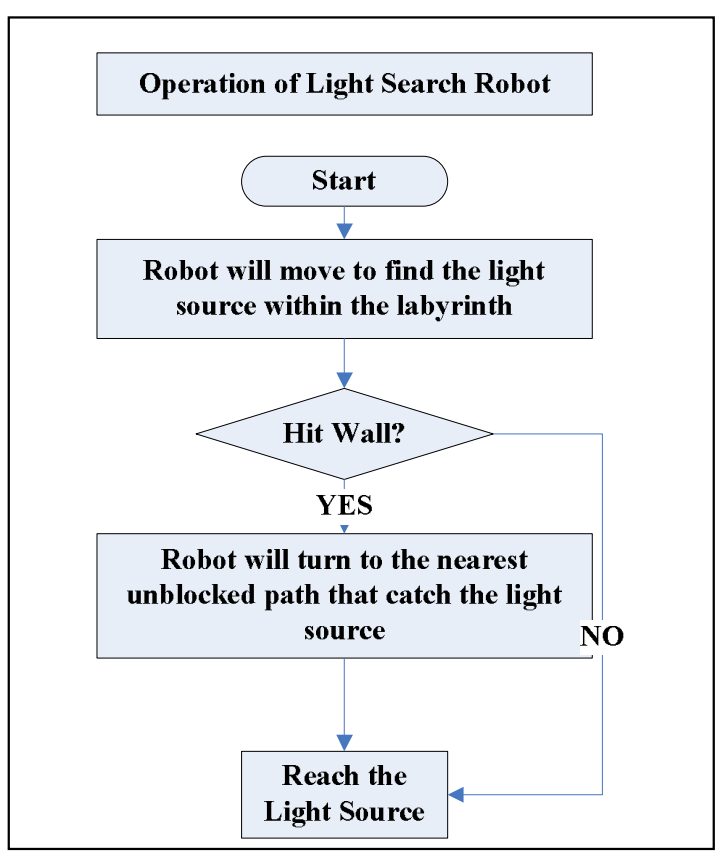

Fig. 6 Operation Mode of Light Search Robot

\section{FuZzy Logic CONTROLler DESIGN}

\section{A. Fuzzy Logic Controller for Line Follower Robot}

The fuzzy truth of the inputs from the two light sensors is divided into 6 fuzzy sets and the output to the two motors is also grouped into 6 fuzzy sets. Although there are many fuzzy logic works that uses the error between the desired and actual output of the sensor as the input the fuzzy logic controller such as in [6], in this project we used the input from the light sensor directly. Table 1 summarizes the fuzzy sets and their respective notations. Trapezoidal and triangular membership functions are used both for the input and output as illustrated in Fig. 7 and Fig. 8 respectively. The input value from the light sensor lie around 80 percent for white colour and 20 percent for black colour. The horizontal scale in Figure 5 represents the percentage reading value from light sensor, and the vertical scale present fuzzy truth value. The system is evaluated based on the fuzzy rule as listed in Table 2 and produces output voltage which directly influences the speed of the right and left motor, that renders the robot to maintain tracking without deviating from its path.

TABLE 1

Input and Output Fuzzy Sets for Line Following Robot

\begin{tabular}{|l|l|}
\hline \multicolumn{1}{|c|}{ Fuzzy Set } & Notation \\
\hline High Input for Right Sensor & ARF_High \\
\hline High Input for Left Sensor & ALF_High \\
\hline Medium Input for Right Sensor & ARF_Med \\
\hline Medium Input for Left Sensor & ALF_Med \\
\hline Low Input for Right Sensor & ARF_Low \\
\hline Low Input for Left Sensor & ALF_Low \\
\hline High Output for Right Sensor & BRF_High \\
\hline High Output for Left Sensor & BLF_High \\
\hline Medium Output for Right Motor & BRF_Med \\
\hline Medium Output for Left Motor & BLF_Med \\
\hline Low Output for Right Motor & BRF_Low \\
\hline Low Output for Left Motor & BLF_Low \\
\hline
\end{tabular}

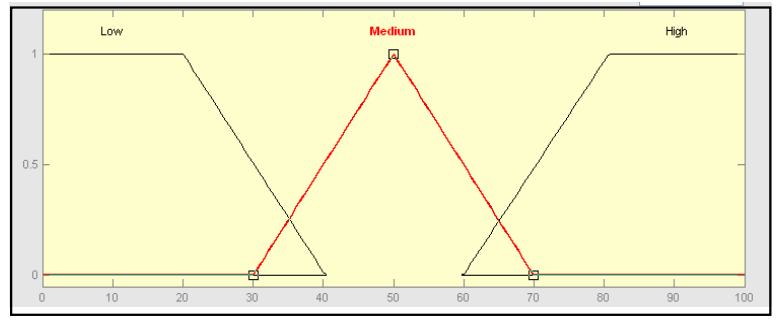

Fig. 7 Fuzzy Logic Membership Function for the Left and Right Light Sensor Input

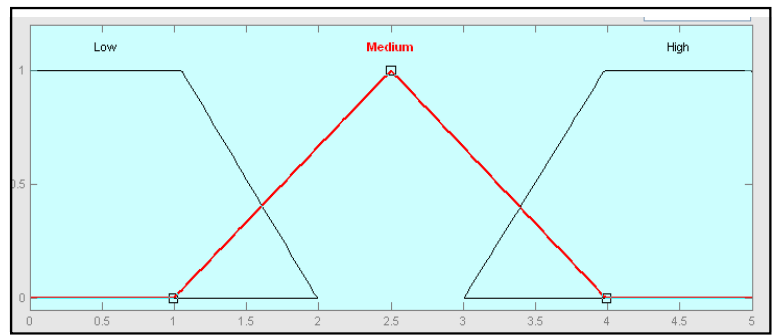

Fig. 8 Fuzzy Logic Membership Function for the Left and Right Motor Output 
TABLE 2

Fuzzy Logic Rule for Line Following Robot

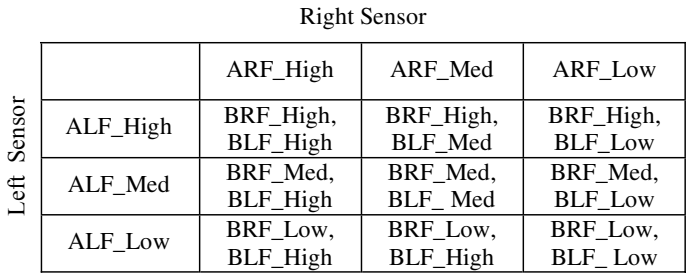

\section{B. Fuzzy Logic Controller for Light Searching Robot}

The fuzzy controlled light searching robot has 6 fuzzy input sets which are the low, medium and high readings from the left and right light sensors. The motor output is also divided into 6 fuzzy sets representing three ranges of left and right motor speed that will be affected by the voltage produced which are low, medium and high. The notations of these fuzzy sets are summarized in Table 3 . The rule set is summarised in Table 4 and is illustrated in the rule viewer as in Fig. 9.

TABLE 3

Input and Output Fuzzy Sets for Light Searching Robot

\begin{tabular}{|l|l|}
\hline \multicolumn{1}{|c|}{ Fuzzy Set } & Notation \\
\hline High Input for Right Sensor & ALR_High \\
\hline High Input for Left Sensor & ALL_High \\
\hline Medium Input for Right Sensor & ALR_Med \\
\hline Medium Input for Left Sensor & ALL_Med \\
\hline Low Input for Right Sensor & ALR_Low \\
\hline Low Input for Left Sensor & ALL_Low \\
\hline High Output for Right Sensor & BLR_High \\
\hline High Output for Left Sensor & BLL_High \\
\hline Medium Output for Right Motor & BLR_Med \\
\hline Medium Output for Left Motor & BLL_Med \\
\hline Low Output for Right Motor & BLR_Low \\
\hline Low Output for Left Motor & BLL_Low \\
\hline
\end{tabular}

TABLE 4

Fuzzy Logic Rule for Light Searching Robot

Right Sensor

\begin{tabular}{|c|c|c|c|}
\hline & ALR_High & ALR_Med & ALR_Low \\
\hline \multirow{2}{*}{ ALL_High } & $\begin{array}{c}\text { BLR_Low, } \\
\text { BLL_Low }\end{array}$ & $\begin{array}{c}\text { BLR_Med, } \\
\text { BLL_High }\end{array}$ & $\begin{array}{c}\text { BLR_Low, } \\
\text { BLL_High }\end{array}$ \\
\hline \multirow{5}{*}{ ALL_Med } & $\begin{array}{c}\text { BLR_High, } \\
\text { BLL_Med }\end{array}$ & $\begin{array}{c}\text { BLR_Med, } \\
\text { BLL_Med }\end{array}$ & $\begin{array}{c}\text { BLR_High, } \\
\text { BLL_Med }\end{array}$ \\
\hline \multirow{2}{*}{ ALL_Low } & $\begin{array}{c}\text { BLR_Med, } \\
\text { BLL_Low }\end{array}$ & $\begin{array}{c}\text { BLR_Med, } \\
\text { BLL_Low }\end{array}$ & $\begin{array}{c}\text { BLR_High, } \\
\text { BLL_High }\end{array}$ \\
\hline
\end{tabular}

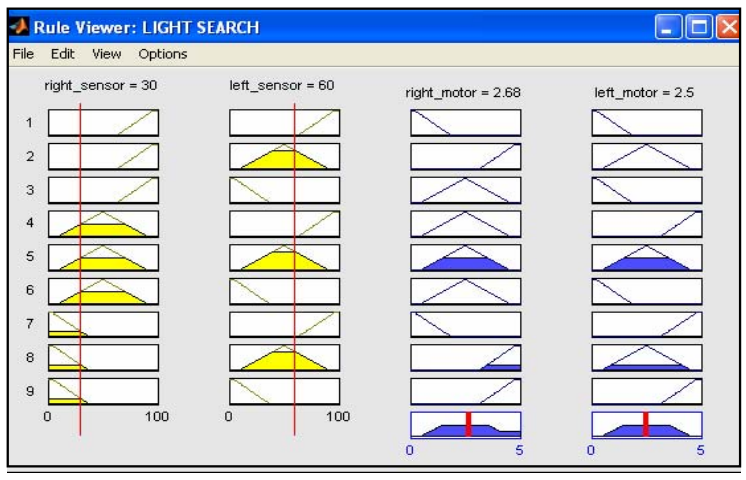

Fig. 9 Rule viewer

\section{EXPERIMENTAL RESULTS AND DISCUSSION}

\section{A. Fuzzy Logic Line Follower Robot and Sorter Robot Experimental Results}

The robot is tested to track a path as shown in Fig. 10, which consists of straight line, curve line, and crossroads. The line following robot is programmed by using the Interactive $\mathrm{C}$ software and the sorter robot is programmed by using the Robolab software. The large advantage of Robolab is that it is very easy to get started programming using it.

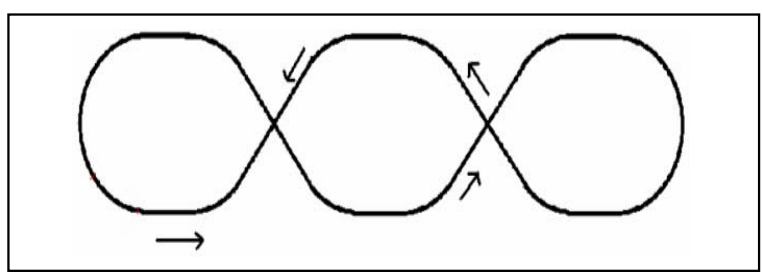

Fig. 10 Line Following Robot Path

A line following robot with binary (on/off) control as in Fig. 11 is developed as a comparison purpose in evaluating the performance of the fuzzy logic controlled robot. The results of the experiment are summarised in Table 5.

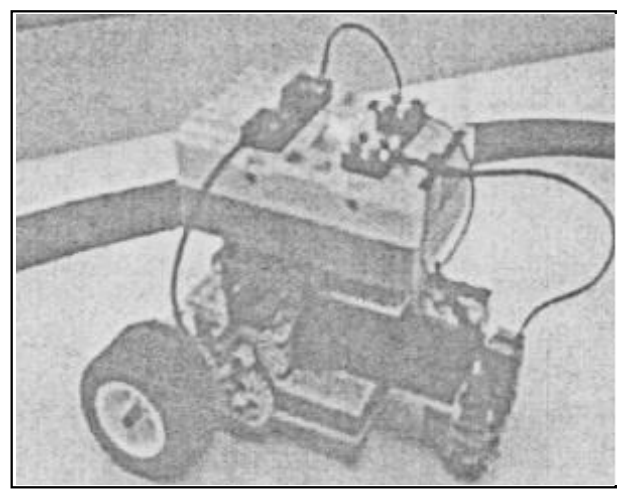

Fig. 11 Binary Line Following Robot TABLE 5 
Experimental Results for Line Following Robot

\begin{tabular}{|l|c|c|}
\hline \multicolumn{1}{|c|}{ Criteria } & $\begin{array}{c}\text { Fuzzy Logic } \\
\text { Controlled Robot }\end{array}$ & Binary Robot \\
\hline $\begin{array}{l}\text { Time to complete one } \\
\text { whole circuit }\end{array}$ & $27.11 \mathrm{~s}$ & $52.51 \mathrm{~s}$ \\
\hline Line tracking & Smooth & Not so smooth \\
\hline Velocity & $0.2 \mathrm{~m} / \mathrm{s}$ & $0.1 \mathrm{~m} / \mathrm{s}$ \\
\hline $\begin{array}{l}\text { Tendency to astray } \\
\text { from line }\end{array}$ & Low & High \\
\hline
\end{tabular}

From the data in the table, it can be observed that fuzzy logic controlled robot has better performance in every criteria listed in the table compared to uncontrolled robot. The fuzzy controlled robot has higher velocity, consumes less time to complete one whole circuit, tracks the line smoother and has lower tendency to astray from line compared to uncontrolled robot. Therefore this system can be used in training and showing the undergraduate students on fuzzy logic control system, its application and implementation in the real world and the advantages that it offers.

\section{B. Light Searching Robot Experimental Results}

To investigate the effectiveness of the proposed approach, the light search robot is tested in a maze, made of black board as shown in Fig. 12. The robot is placed at the starting point and a lamp which acts as the light source is positioned in the maze.

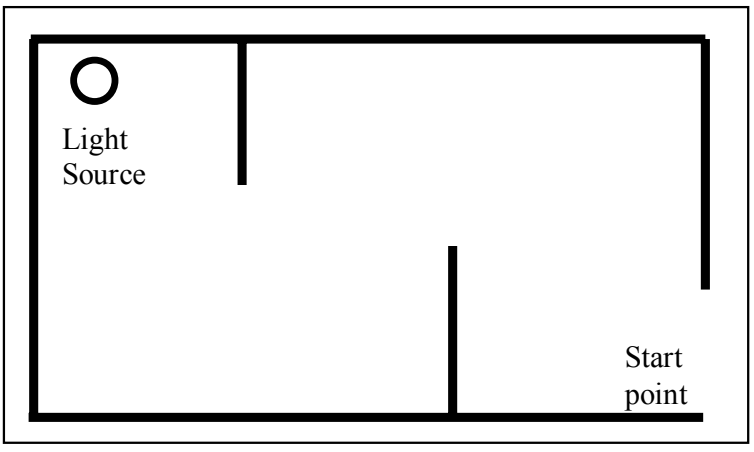

Fig. 12 Maze Challenge

As a comparison, a nonfuzzy robot is developed and tested in the same maze to evaluate the performance of the fuzzy logic controlled robot. From the experimental results, it can be seen that the fuzzy robot is more efficient in detecting and moving towards the light source without colliding with the walls within a shorter period. On the other hand, although the nonfuzzy robot finally successful to track the light source, it takes a comparatively longer time to accomplish the task as it tends to encircle around the maze and deviates from the path after sometimes. The efficiency of the light searching fuzzy robot and the inefficiency of its nonfuzzy counterpart can be used to demonstrate the concepts, real world applications and necessity of fuzzy logic to motivate and help the students to understand the subject better.

\section{CONCLUSION}

A fuzzy logic training system based on line following and light searching robot for undergraduate fuzzy logic class is presented in this paper. The fuzzy logic controlled robot is shown to have better performance compared to uncontrolled robot. With this training system, students will be highly motivated and able to understand the concept of fuzzy logic better. They will not only learn the subject theoretically in the class, but also has the experience on the real implementation on fuzzy logic control.

\section{REFERENCES}

[1] Timothy J. Ross, Fuzzy Logic with Engineering Applications, Mc Graw Hill, 2001.

[2] Eric Wang, "Teaching Freshman Design, Creativity and Programming with Legos and Labview", Reno, $33^{s t}$ ASEE/IEEE Frontiers in Education Conference, 2001, pp. 11-15.

[3] R. F. Reuss, T. M. Lee, "Fuzzy Logic Control in a Line Following Robot", Class Notes of University of Nevada at Reno, Nevada,, 2001.

[4] Carl Looney, "Class Notes for CS791j”, Nevada, 2001.

[5] Paul Wallich, "Mindstorms Not Just a Kid's Toy", IEEE Spectrum, 2001, pp. 52- 57.

[6] Marley Maria B.R. Vellasco, Marco Aurelio C. Pacheo, Ivo Lima Brasil Jr., "Mobile Robot Control Using Fuzzy Logic", 1st International Khepera Workshop (IKW99), Brazil, 1999. 